\title{
THE POINCARÉ METHOD FOR AN OSCILLATOR WITH QUADRATIC NONLINEARITY
}

\author{
Nguyen Van Dinh and Tran Duong Tri \\ Institute of Mechanics \\ Vietnamese Academy of Science and Technology
}

In [2], to evaluate free oscillation period of an undamped oscillator with large cubic restoring nonlinearity, a modified Poincaré method has been proposed. There, in the neighbourhood of the free oscillation of interest, the strongly nonlinear system under consideration is assumed to be near certain linear one with unknown (to be evaluated) frequency.

In the present paper, we deal with the case of quadratic non-linearity. An additional modification is introduced consisting in the elimination of constant derivation and second harmonic terms. The results obtained show that the domain of application of the Poincaré method can be enlarged.

\section{Systems under consideration and exact free oscillation pe- riod}

Consider an oscillator described by the differential equation

$$
\ddot{x}+x+\beta x^{2}=0,
$$

where $\beta>0$ is coefficient of quadratic nonlinearity; other notations retain their significations explained in [2]. In the phase plane $O x \dot{x}$, the origin $O(0,0)$ and the point $I\left(\frac{-1}{\beta}, 0\right)$ represent two equilibrium states: the first one is a stable center, the second one is a saddle. The domain where oscillations occur contains the center $O$ and is bounded by an homoclinic orbit IJI starting from and ending at the same point $I$, intersecting the axis $O x$ at the point $J\left(\frac{1}{2 \beta}, 0\right)$. If $\beta$ is very small, the mentioned oscillatory domain is very large and the standard Poincaré method is applicable. Contrarily, if $\beta$ is very large, the oscillatory domain enclosed by the homoclinic orbit IJI is very small and the problem of oscillation becomes insignificant. So, below, we are interested only in the case in which the quadratic nonlinearity coefficient $\beta$ is of "medium magnitude" - not too small and also not too large.

The system under consideration is autonomous then, initial conditions for free oscillations can be chosen as:

$$
x(0)=a_{0}>0, \quad \dot{x}(0)=0 .
$$


Obviously, on $a_{0}$, the following requirement is imposed

$$
a_{0}<\frac{1}{2 \beta}=\frac{0.5}{\beta} \quad\left(\text { for } \beta=1, a_{0}<0.5\right) .
$$

The potential energy has as expression

$$
V(x)=\frac{x^{2}}{2}+\beta \frac{x^{3}}{3} .
$$

By $x_{2}, x_{1}, a_{0}\left(x_{2}<-\frac{1}{\beta}<x_{1}<0<a_{0}<\frac{1}{2 \beta}\right)$ we denote three roots of the equation

$$
V\left(a_{0}\right)-V(x)=0
$$

The exact period of the free oscillation satisfying the initial condition (1.2) is given by the integral [3]

$$
T_{e x}=\sqrt{2} \int_{x_{1}}^{a_{0}} \frac{d x}{\sqrt{V\left(x_{0}\right)-V(x)}} .
$$

\section{Period from the standard Poincaré method}

For the sake of comparison, the standard Poincare method is first used to examine the case of small $\beta$.

Being small, $\beta$ can be labeled by a formal small parameter $\varepsilon$; so, the differential equation (1.1) can be written as:

$$
\ddot{x}+x=-\varepsilon \beta x^{2} .
$$

Let $\omega=2 \pi / T$ be the unknown frequency of the free oscillation satisfying the initial conditions (1.2). By introducing the dimensionless time $\tau=\omega t$, the equation (2.1) becomes:

$$
\omega^{2} x^{\prime \prime}+x=-\varepsilon \beta x^{2}
$$

where primes denote differentiation with respect to $\tau$.

Then both unknowns $x$ and $\omega$ are expanded in powers of $\varepsilon$, that is

$$
\begin{aligned}
x & =x_{0}(\tau)+\varepsilon x_{1}(\tau)+\varepsilon^{2} x_{2}(\tau)+\ldots, \\
\omega & =1+\varepsilon \omega_{1}+\varepsilon^{2} \omega_{2}+\ldots
\end{aligned}
$$

Corresponding to $(1.2)$, the initial conditions of $x_{0}(\tau), x_{i}(\tau)$ are:

$$
\begin{aligned}
& x_{0}(\tau=0)=a_{0}, \quad x_{0}^{\prime}(\tau=0)=0, \\
& x_{i}(\tau=0)=0, \quad x_{i}^{\prime}(\tau=0)=0, \quad(i=1,2, \ldots) .
\end{aligned}
$$


Substituting (2.3), (2.4) into (2.2), equating coefficients of like power of $\varepsilon$ yield:

$$
\begin{aligned}
& x_{0}^{\prime \prime}+x_{0}=0, \\
& x_{1}^{\prime \prime}+x_{1}=-2 \omega_{1} x_{0}^{\prime \prime}-\beta x_{0}^{2}, \\
& x_{2}^{\prime \prime}+x_{2}=-2 \omega_{1} x_{1}^{\prime \prime}-\left(\omega_{1}^{2}+2 \omega_{2}\right) x_{0}^{\prime \prime}-2 \beta x_{0} x_{1},
\end{aligned}
$$

With regard to initial conditions (2.5), the solution $x_{0}$ is

$$
x_{0}=a_{0} \cos \tau .
$$

Using (2.10) we rewrite (2.8) as:

$$
x_{1}^{\prime \prime}+x_{1}=2 \omega_{1} a_{0} \cos \tau-\frac{1}{2} \beta a_{0}^{2}(1+\cos 2 \tau) .
$$

To eliminate the secular term $2 \omega_{1} a_{0} \cos \tau$ in (2.11), the additional frequency $\omega_{1}$ should be taken:

$$
\omega_{1}=0 .
$$

Then the solution $x_{1}$ satisfying the initial conditions (2.6) is:

$$
x_{1}=-\frac{1}{2} \beta a_{0}^{2}(1-\cos \tau)+\frac{1}{6} p a_{0}^{2}(\cos 2 \tau-\cos \tau) .
$$

With regard to $(2.10),(2.12),(2.13)$, the differential equation (2.9) can be written as:

$$
\begin{aligned}
x_{2}^{\prime \prime}+x_{2} & =2 \omega_{2} a_{0} \cos \tau-2 \beta^{2} a_{0}^{3} \cos \tau\left\{-\frac{1}{2}+\frac{1}{3} \cos \tau+\frac{1}{6} \cos 2 \tau\right\} \\
& =-\frac{1}{3} \beta a_{0}^{3}+\left(2 \omega_{2} \frac{5}{6} \beta a_{0}^{2}\right) a_{0} \cos \tau-\frac{1}{3} a_{0}^{3} \cos 2 \tau-\frac{1}{6} \beta^{2} a_{0}^{3} \cos 3 \tau .
\end{aligned}
$$

Eliminating secular term yields:

$$
\omega_{2}=-\frac{5}{12} \beta^{2} a_{0}^{2} .
$$

Thus, in the second approximation, the formulas for frequency and period are, respectively:

$$
\omega=1-\frac{5}{12} \beta^{2} a_{0}^{2}, \quad T_{0}=\frac{2 \pi}{\left(1-\frac{5}{12} \beta^{2} a_{0}^{2}\right)} .
$$

Note that the condition $\omega>0$ requies

$$
a_{0}<\frac{1}{\beta} \sqrt{\frac{12}{5}} \approx \frac{1.5492}{\beta} .
$$

which differs enough from (1.3). This means that the range of validity of the standard Poincare method is limited and can only be extended by suitable modifications. 


\section{Period from a modified Poincaré method}

In this section, with additional modification, the modified Poincaré method proposed in $[2]$ is applied to treat the case in which $\beta$ is not too small.

The differential equation (1.1) is rearranged as:

$$
\ddot{x}+\omega^{2} x=\left(\omega^{2}-1\right) x-\beta x^{2},
$$

or, by using the dimensionless time $\tau=\omega t$

$$
\omega^{2}\left(x^{\prime \prime}+x\right)=\left(\omega^{2}-1\right) x-\beta x^{2},
$$

where $\omega$ is the unknown (to be evaluaed) frequency of the free oscillation satisfying the initial conditions (1.2).

Note that for $x=a \cos \tau$, the quadratic nonlinearity $\beta x^{2}$ "produces" the derivation $\frac{1}{2} \beta a^{2}$ and also the second harmonic $\frac{1}{2} \beta a^{2} \cos 2 \tau$; these two components may be large enough and could not be "neutralized" by the first harmonic $\left(\omega^{2}-1\right) a \cos \tau$. Therefore, the right hand side of (3.2) should not be small and the differential equation (3.2) could not be considered as the one belonging to weakly nonlinear type.

Let us decompose $x$ into two parts, that is

$$
x=y+z
$$

and rewrite (3.2) in the form:

$$
\omega^{2}\left(y^{\prime \prime}+y\right)=\mu\left\{-\omega^{2}\left(z^{\prime \prime}+z\right)+\left(\omega^{2}-1\right)(y+z)-\beta(y+z)^{2}\right\},
$$

where $z$ should be chosen in such a way that the right hand side of (3.4) may be labeled by a new small parameter $\mu$.

The three unknowns $\omega, y, z$ are then expanded in power of $\mu$, that is:

$$
\begin{aligned}
\omega^{2} & =\omega_{0}^{2}+\mu \omega_{1}+\mu^{2} \omega_{2}+\ldots, \\
y & =y_{0}+\mu y_{1}+\mu^{2} y_{2}+\ldots \\
z & =z_{0}+\mu z_{1}+\mu^{2} z_{2}+\ldots
\end{aligned}
$$

The initial conditions (1.2) can be written in the form:

$$
\begin{aligned}
y_{0}(0) & =a, \quad y_{0}^{\prime}(0)=0, \\
y_{i}(0) & =0, \quad y_{i}^{\prime}(0)=0, \\
z_{0}^{\prime}(0) & =0, \quad z_{i}^{\prime}(0)=0, \\
a_{0} & =a+\mu z_{1}(0)+\mu^{2} z_{2}(0)+\ldots
\end{aligned}
$$

Substituting (3.5), (3.6), (3.7) into (3.4), equating the terms of like power of $\mu$ yield:

$$
\begin{aligned}
\omega_{0}^{2}\left(y_{0}^{\prime \prime}+y_{0}\right)=0 & \\
\omega_{0}^{2}\left(y_{1}^{\prime \prime}+y_{1}\right)= & -\omega_{0}^{2} z_{0}^{\prime \prime}-z_{0}+\left(\omega_{0}^{2}-1\right) y_{0}-\beta\left(y_{0}+z_{0}\right)^{2}, \\
\omega_{0}^{2}\left(y_{2}^{\prime \prime}+y_{2}\right)= & -\omega_{1}\left(y_{1}^{\prime \prime}+y_{1}\right)-\omega_{0}^{2} z_{1}^{\prime \prime}-\omega_{1} z_{0}^{\prime \prime}-z_{1} \\
& +\left(\omega_{0}^{2}-1\right) y_{1}+\omega_{1} y_{0}-2 \beta\left(y_{0}+z_{0}\right)\left(y_{1}+z_{1}\right),
\end{aligned}
$$


The solution $y_{0}$ satisfying the initial condition $(\dot{3} .8)$ is

$$
y_{0}=a \cos \tau \text {. }
$$

Using (3.15) and choosing

$$
z_{0}=b_{0}+c_{0} \cos 2 \tau
$$

the differential equation (3.13) becomes:

$$
\begin{aligned}
\omega_{0}^{2}\left(y_{1}^{\prime \prime}+y_{1}\right)= & \left(\omega_{0}^{2}-1\right) a \cos \tau-b_{0}+\left(4 \omega_{0}^{2}-1\right) c_{0} \cos 2 \tau-\beta a^{2} \cos ^{2} \tau-2 \beta a b_{0} \cos \tau \\
& \quad-2 \beta a c_{0} \cos \tau \cos 2 \tau-\beta b_{0}^{2}-2 \beta b_{0} c_{0} \cos 2 \tau-\beta c_{0}^{2} \cos ^{2} 2 \tau \\
= & \left(\omega_{0}^{2}-1\right) a \cos \tau-b_{0}+\left(4 \omega_{0}^{2}-1\right) c_{0} \cos 2 \tau-\frac{1}{2} \beta a^{2}(1+\cos 2 \tau) \\
& -2 \beta a b_{0} \cos \tau-\beta a c_{0}(\cos 3 \tau+\cos \tau)-\beta b_{0}^{2} \\
& -2 \beta b_{0} c_{0} \cos 2 \tau-\frac{1}{2} \beta c_{0}^{2}(1+\cos 4 \tau) .
\end{aligned}
$$

Eliminating secular terms and also constant and second harmonic terms yields:

$$
\begin{aligned}
& \left(\omega_{0}^{2}-1\right)-2 \beta b_{0}-\beta c_{0}=0, \\
& b_{0}+\frac{1}{2} \beta a^{2}+\beta b_{0}^{2}+\frac{1}{2} \beta c_{0}^{2}=0, \\
& \left(4 \omega_{0}^{2}-1\right) c_{0}-\frac{1}{2} \beta a^{2}-2 \beta b_{0} c_{0}=0 .
\end{aligned}
$$

From (3.18), it follows:

$$
c_{0}=\frac{1}{\beta}\left(\omega_{0}^{2}-1-2 \beta b_{0}\right) .
$$

Substituting (3.21) into (3.19), (3.20) gives:

$$
\begin{aligned}
& 6 \beta^{2} b_{0}^{2}+\left(6-4 \omega_{0}^{2}\right) \beta b_{0}+\left(\omega_{0}^{2}-1\right)^{2}+\beta^{2} a^{2}=0, \\
& 8 \beta^{2} b_{0}^{2}+\left(8-20 \omega_{0}^{2}\right) \beta b_{0}+8 \omega_{0}^{4}-10 \omega_{0}^{2}+2-\beta^{2} a^{2}=0,
\end{aligned}
$$

from which we can deduce

$$
\beta_{0} \doteq \frac{1}{44 \omega_{0}^{2}}\left(20 \omega_{0}^{4}-22 \omega_{0}^{2}+\left(2-7 \beta^{2} a^{2}\right)\right) .
$$

With regard to (3.24), the equation (3.23) can be transformed into the form:

$$
136 \omega_{0}^{8}-\left(140-248 \beta^{2} a^{2}\right) \omega_{0}^{4}+\left(2-7 \beta^{2} a^{2}\right)^{2}=0 .
$$

The formulae for $\omega_{0}^{4}$ is given by the positive solution satisfying the condition $\left.\omega_{0}\right|_{\beta=0}=1$, that is

$$
\omega_{0}^{4}=\frac{1}{68}\left\{\left(35-62 \beta^{2} a^{2}\right)+\sqrt{\left(35-62 \beta^{2} a^{2}\right)^{2}-34\left(2-7 \beta^{2} a^{2}\right)^{2}}\right\} .
$$


The existence of this root requires:

$$
35-62 \beta^{2} a^{2}>0, \quad\left(35-62 \beta^{2} a^{2}\right)^{2}-34\left(2-7 \beta^{2} a^{2}\right)^{2} \geq 0,
$$

which leads to the inequality:

$$
\beta a \leq 0.6982 \text { or } a<\frac{0.6982}{\beta} .
$$

Although (3.28) is still unexact, it is better than $(2.17)$ : for $\beta=1$, the value $a \approx 0.65$ corresponds to the limit value $a_{0}=0.5$.

The differential equation governing $y_{1}$ is rather simple

$$
\omega_{0}^{2}\left(y_{1}^{\prime \prime}+y_{1}\right)=-\beta a c_{0} \cos 3 \tau-\frac{1}{2} \beta c_{0}^{2} \cos 4 \tau,
$$

and the solution $y_{1}$ satisfying the initial conditions $(3.9)$ is:

$$
y_{1}=\frac{\beta a c_{0}}{8 \omega_{0}^{2}}(\cos 3 \tau-\cos \tau)+\frac{\beta c_{0}^{2}}{30 \omega_{0}^{2}}(\cos 4 \tau-\cos \tau) .
$$

Going on the presented procedure, by choosing $z_{1}=b_{1}+c_{1} \cos 2 \tau$, three linear algebraic equations for determining $\omega_{1}, b_{1}, c_{1}$ are obtained

$$
\begin{aligned}
& \left(1+2 \beta b_{0}\right) b_{1}+\left(\beta c_{0}\right) c_{1}=\frac{\beta^{2} c_{0} a^{2}}{8 \omega_{0}^{2}}+\frac{\beta^{2} c_{0}^{2} a}{30 \omega_{0}^{2}} \\
& -\left(2 \beta c_{0}\right) b_{1}+\left(4 \omega_{0}^{2}-1-2 \beta b_{0}\right) c_{1}+\left(8 \omega_{0} c_{0}\right) \omega_{1}=\frac{\beta^{2} c_{0}^{3}}{30 \omega_{0}^{2}}-\frac{\beta^{2} c_{0}^{2} a}{30 \omega_{0}^{2}}, \\
& (2 \beta a) b_{1}+(\beta a) c_{1}-\left(2 \omega_{0} a\right) \omega_{1}=\frac{\beta^{2} b_{0} c_{0} a}{4 \omega_{0}^{2}}+\frac{\beta^{2} b_{0} c_{0}^{2}}{15 \omega_{0}^{2}}+\frac{\beta^{2} c_{0}^{3}}{30 \omega_{0}^{2}}-\left(\omega_{0}^{2}-1\right)\left(\frac{\beta c_{0} a}{8 \omega_{0}^{2}}+\frac{\beta c_{0}^{2}}{30 \omega_{0}^{2}}\right) .
\end{aligned}
$$

Thus, in the second approximation, the period of the free oscillation satisfying the initial condition (1.2) is given by the formulae

$$
a_{0}=a+b_{0}+c_{0}+b_{1}+c_{1}, \quad T=\frac{2 \pi}{\omega}=\frac{2 \pi}{\omega_{0}+\omega_{1}} .
$$

Remark. Instead of the form (3.8)-(3.11), the initial condition (1.2) can be written as:

$$
\begin{aligned}
& y_{0}(0)=a_{0}, \quad y_{0}^{\prime}(0)=0, \quad y_{i}(0)=y_{i}^{\prime}(0)=0, \\
& z_{0}(0)=z_{i}(0)=z_{0}^{\prime}(0)=z_{i}^{\prime}(0)=0, \quad(i=1,2, \ldots) .
\end{aligned}
$$

In this case, the form of $z_{0}$ is:

$$
z_{0}=b_{0}(1-\cos \tau)+c_{0}(\cos 2 \tau-\cos \tau)
$$

However, the estimation (3.28) cannot be obtained. 


\section{Numerical results}

For $\beta=1$ (the limit value of the initial position is 0.5 ) numerical results obtained are shown in the following Table

\begin{tabular}{|c|c|c|c|c|}
\hline$a$ & $a_{0}=a+b_{0}+c_{0}+b_{1}+c_{1}$ & $T_{e x}$ & $T_{0}$ & $T$ \\
\hline 0.00 & 0.0000 & 6.2832 & 6.2832 & 6.2832 \\
\hline 0.02 & 0.0399 & 6.2842 & 6.2842 & 6.2842 \\
\hline 0.04 & 0.0395 & 6.2873 & 6.2873 & 6.2874 \\
\hline 0.06 & 0.0588 & 6.2926 & 6.2922 & 6.2926 \\
\hline 0.08 & 0.0799 & 6.3000 & 6.2991 & 6.3001 \\
\hline 0.10 & 0.0967 & 6.3096 & 6.3077 & 6.3096 \\
\hline 0.20 & 0.1865 & 6.3925 & 6.3756 & 6.3926 \\
\hline 0.30 & 0.2690 & 6.5438 & 6.4786 & 6.5441 \\
\hline 0.40 & 0.3434 & 6.7905 & 6.6080 & 6.7913 \\
\hline 0.50 & 0.4048 & 7.1942 & 6.7524 & 7.1954 \\
\hline 0.60 & 0.4631 & 7.9780 & 6.8997 & 7.9181 \\
\hline 0.61 & 0.4684 & 8.1201 & 6.9154 & 8.0201 \\
\hline 0.62 & 0.4740 & 8.3006 & 6.9322 & 8.1279 \\
\hline 0.63 & 0.4802 & 8.5563 & 6.5912 & 8.2391 \\
\hline 0.64 & 0.4877 & 9.0101 & 6.9745 & 8.3469 \\
\hline 0.65 & 0.4892 & 10.9180 & 7.0080 & 8.4267 \\
\hline
\end{tabular}

For relative error of order $4 \%$, the period $T$ from the modified Poincare method is acceptable if $a_{0} \leq 0.48$ (about $96 \%$ of the oscillatory domain) while the standard one is acceptable only if $a_{0} \leq 0.4$ (about $80 \%$ ). The domain of application of the standard Poincaré method is not too narrow; this results from the fact that the quadratic nonlinearity is not very strong and the oscillatory domain is limited

\section{Conclusion}

An oscillator with not too small quadratic nonlinearity is considered. The governing differential equation is written as for weakly nonlinear oscillator after eliminating the deviation and second harmonic terms. The results obtained can be used for a great part of the oscillatory domain.

This publication is completed with the financial support from The Council for Natural Science of Vietnam.

\section{References}

1. Nayfeh A. N., Pertubation Method, Wiley, New York, 1973.

2. Nguyen Van Dinh, The Poincaré method for a strongly nonlinear Duffing oscillator, Vietnam Journal of Mechanics 25 (2003) 19-25. 
3. Kauderer H., Nichtlineare Mechanic, Springer Verlag, Berlin, 1958.

Received July 8, 2003

\section{PHƯONG PHÁP POINCARÉ CHO CHẤN TỬ VỚI PHI TUYẾN BẬC HAI}

Xét chấn tử không có cản, không bị kích động, có phi tuyến hồi phục bậc hai không quá nhỏ. Phương trình vi phân dao động được viết như với chấn tử phi tuyến yếu sau khi loại bỏ độ lệch và ác-mô-nic thứ hai, kết quả tính chu kỳ dao động tự do có thể chấp nhận với phần lớn miền dao động. 